\title{
Une méthode de pénalité-projection pour les écoulements dilatables
}

\author{
Matthieu Jobelin* — Bruno Piar* — Philippe Angot** \\ Jean-Claude Latché* \\ * Institut de Radioprotection et de Sûreté Nucléaire (IRSN) \\ BP 3, F-13115 Saint Paul Lez Durance cedex \\ ** Université de Provence et Laboratoire d'Analyse, Topologie et Probabilités \\ (LATP), 39 rue F. Joliot Curie, F-13453 Marseille cedex 13
}

\begin{abstract}
RÉSUMÉ. Nous présentons dans cet article une nouvelle méthode de correction de pression pour les écoulements dilatables. Nommée "méthode de pénalité-projection », cette technique diffère des schémas de projection usuels par l'ajout dans l'étape de prédiction d'un terme de pénalisation, construit pour contraindre la vitesse à satisfaire le bilan de masse. Ce terme est multiplié par un coefficient $\mathrm{r}$, dit paramètre de pénalisation. Nous montrons par des expériences numériques que ce schéma est bien plus précis que la méthode usuelle. L'erreur de fractionnement, dominante à fort pas de temps, est réduite à volonté en augmentant $\mathrm{r} ; \grave{a}$ noter, toutefois, que l'usage d'une valeur trop importante dégrade le conditionnement de l'opérateur associé à l'étape de prédiction. Par ailleurs, les pertes de convergence de la méthode de projection usuelle en cas de conditions aux limites ouvertes sont corrigées, dès que r est non nul.

ABSTRACT. We present in this paper a novel pressure correction method for dilatable flows. Named "penalty-projection method", this scheme differs from the usual projection method by the addition in the prediction step of a penalty term, built to enforce the mass balance. This term is proportional to a penalty parameter $\mathrm{r}$. Numerical experiments show that the accuracy of the scheme is drastically improved. The splitting error, dominant at large time steps, may be reduced down to zero by choosing larger and larger values for $\mathrm{r}$; note, however, that a too large value degrades the conditioning of the operator associated to the prediction step. In addition, the loss of convergence observed with the standard projection method in case of open boundary conditions is not observed anymore, as soon as $\mathrm{r}$ is nonzero.

MOTS-CLÉS : écoulements dilatables, écoulements à faible nombre de Mach, méthode de projection, méthode de pénalité, méthodes à pas fractionnaire.
\end{abstract}

KEYWORDS: dilatable flows, low Mach number flows, projection method, penalty method.

DOI:10.3166/REMN.17.453-480 @ 2008 Lavoisier, Paris 


\section{Introduction}

La modélisation d'une grande variété d'écoulements physiques conduit à des représentations mathématiques où vient s'associer aux équations de bilan de masse et de quantité de mouvement une équation de transport-diffusion d'une variable additionnelle, $z$, dont la masse volumique se déduit :

$$
\mid \begin{array}{lr}
\frac{\partial \varrho z}{\partial t}+\nabla \cdot \varrho z u=\nabla \cdot \mathcal{D} \nabla z & \text { dans }[0, T] \times \Omega \\
\varrho=\mathcal{G}(z) & \\
\frac{\partial \varrho u}{\partial t}+\nabla \cdot(\varrho u \otimes u)=\nabla \cdot \tau(u)-\nabla p+f & \text { dans }[0, T] \times \Omega \\
\frac{\partial \varrho}{\partial t}+\nabla \cdot \varrho u=0 & \text { dans }[0, T] \times \Omega
\end{array}
$$

La variable $\varrho$ désigne ici la masse volumique, $t$ le temps, $u$ la vitesse, $p$ la pression, $f$ une force volumique répartie, $\mathcal{D}$ est un coefficient de diffusion et $\tau$ est le tenseur des contraintes visqueuses. L'ensemble $\Omega$ est un domaine régulier de $\mathbb{R}^{d}, d=2$ ou $d=3$ et $T$ est le temps final, $T<\infty$. Pour fixer les idées, on peut voir la fonction $\mathcal{G}(\cdot)$ comme une loi d'état. Pour que le problème soit complètement défini, ce système doit être complété par des conditions aux limites et initiales.

Les problèmes de convection naturelle à faible nombre de Mach, par exemple, rentrent dans ce formalisme, lorsqu'ils sont traités en utilisant un modèle asymptotique, i.e. un système d'équations vérifié par les champs de vitesse et pression dans l'écoulement lorsque le nombre de Mach tend vers zéro, tel que décrit dans (Majda et $a l ., 1985)$. La variable $z$ représente alors la température et la loi $\mathcal{G}(\cdot)$ est déduite de la loi d'état, moyennant le calcul préalable de la pression thermodynamique lorsque le système physique considéré est clos. Si maintenant on identifie $z$ à une concentration et $\varrho=\mathcal{G}(z)$ à une loi de mélange, on obtient les équations de la convection solutale. De la même manière, certains problèmes très simplifiés de combustion s'écrivent sous la forme du système [1]; la variable $z$ prend alors la signification d'une variable d'avancement (Babik et al., 2005). Ces problématiques physiques font partie des phénomènes d'intérêt dans le domaine de la sûreté nucléaire, telles que traitées à l'Institut de Radioprotection et de Sûreté Nucléaire (IRSN); c'est le cadre de la présente étude.

Du fait que la masse volumique du fluide est supposée indépendante de la pression (dite pression dynamique), cette dernière joue d'un point de vue mathématique un rôle similaire à celui qu'elle tient dans les équations de Navier-Stokes incompressibles. Pour s'en convaincre, il suffit de réécrire l'équation de bilan de quantité de mouvement en prenant comme variable le débit massique $q=\varrho u$ et les deux dernières équations du système [1] retrouvent la structure classique d'un problème mixte.

En conséquence, il est naturel de mettre en œuvre pour la résolution numérique de ce problème des schémas initialement développés dans le contexte des écoulements incompressibles. Parmi ceux-ci, les méthodes de projection ont, depuis les travaux originels (Chorin, 1968; Temam, 1969), acquis une popularité croissante. Ce succès tient 
dans le fait que ces schémas à pas fractionnaires découplent à chaque pas de temps les équations de bilan de quantité de mouvement et de bilan de masse, substituant ainsi à un problème mixte, de résolution couplée difficile et coûteuse, une succession de problèmes elliptiques plus aisés à résoudre.

Le principe des méthodes de projection est le suivant. Dans une première étape, on obtient une prédiction de la vitesse par la résolution de l'équation de bilan de quantité de mouvement, dans laquelle la pression est ignorée (méthode originelle) ou approchée par une formule explicite (méthode dite incrémentale). La seconde étape consiste à projeter la vitesse prédite dans l'espace des fonctions à divergence nulle ; cette étape s'apparente à un problème de Darcy, qui est classiquement réécrit comme un problème elliptique pour la pression (méthode originelle) ou pour l'incrément de pression (méthode incrémentale). Sur cette idée de base sont venues, au fil des années, se greffer de multiples variantes. La méthode de projection incrémentale semble avoir été proposée dans (Goda, 1979), le premier schéma formellement de second ordre en temps dans (Van Kan, 1986). Dans l'étape de projection, les conditions aux limites appliquées à l'incrément de pression sont artificielles, i.e. ne sont pas vérifiées par la solution du problème, ce qui induit des pertes de précision, particulièrement graves pour les écoulements visqueux obéissant à des conditions aux limites ouvertes sur une partie de la frontière (Guermond et al., 2005). Ce phénomène est corrigé dans une variante proposée dans (Timmermans et al., 1996) puis analysée dans (Guermond et al., 2003), qui a reçu le nom de méthode rotationnelle. On trouvera une revue de ces différents schémas et de leurs propriétés de convergence respectives dans (Guermond et al., 2006).

Si le découplage des équations de bilan de quantité de mouvement et de bilan de masse simplifie la résolution, il introduit également une erreur numérique, dite erreur de fractionnement, qui devient, pour des discrétisations en temps d'ordre deux, importante voire dominante à fort pas de temps (Jobelin et al., 2006). Cette erreur de fractionnement disparaîtrait si, par un choix judicieux de la pression approchée, la vitesse prédite vérifiait la contrainte de divergence : la vitesse prédite serait alors la même que celle obtenue par un schéma couplé, et l'étape de projection serait sans objet. Bien sûr, un telle estimation de vitesse n'est pas accessible dans la pratique. On peut toutefois s'en approcher en ajoutant dans la première étape un terme de pénalisation associé à la contrainte de divergence, analogue à celui utilisé dans les techniques de lagrangien augmenté : c'est le principe des méthodes de pénalité-projection. Le premier schéma de ce type semble avoir été suggéré dans (Shen, 1992, section 6), puis mis en œuvre indépendamment dans (Caltagirone et al., 1999), dans leur méthode dite de «projection vectorielle». Cette dernière, qui s'appuie sur une discrétisation spatiale en volumes finis et utilise une étape de projection non conventionnelle, est l'un des ingrédients essentiels des schémas du code Aquilon (Aquilon, 2006). Enfin, l'application de ces idées dans le contexte des éléments finis conduit à un schéma original expérimenté dans (Jobelin et al., 2006) et analysé dans (Angot et al., 2007).

Dans cet article, nous étendons ces derniers travaux à des écoulements à masse volumique variable; plus précisément, nous traitons le développement d'une méthode 
de pénalité-projection pour la résolution des équations de bilan de quantité de mouvement et de masse (deux dernières relations du système [1]). On trouvera facilement dans la littérature, et notamment dans (Coré et al., 2002; Coré et al., 2003; Babik et al., 2005; Jobelin, 2006), des exemples de schémas à pas fractionnaires pour des systèmes de la forme [1] faisant apparaître ce problème réduit : lors des étapes de résolution des équations de bilan de masse et de quantité de mouvement, la masse volumique y est en effet connue, du fait que la ou les équation(s) portant sur les variables d'état entrant dans le calcul de cette quantité ont été préalablement résolues.

Le problème ici étudié est ainsi le suivant :

$$
\mid \begin{array}{ll}
\frac{\partial \varrho u}{\partial t}+\nabla \cdot(\varrho u \otimes u)=\nabla \cdot \tau(u)-\nabla p+f & \text { dans }[0, T] \times \Omega \\
\nabla \cdot \varrho u+\frac{\partial \varrho}{\partial t}=0 & \text { dans }[0, T] \times \Omega \\
u=u_{\mathrm{D}} & \operatorname{sur}[0, T] \times \Gamma_{\mathrm{D}} \\
-p n+\tau(u) \cdot n=g_{\mathrm{N}} & \text { sur }[0, T] \times \Gamma_{\mathrm{N}}
\end{array}
$$

où $\varrho=\varrho(x, t)$ est une fonction régulière donnée.

Les surfaces $\Gamma_{\mathrm{D}}$ et $\Gamma_{\mathrm{N}}$ forment une partition de la frontière $\Gamma$ du domaine de calcul de normale extérieure $n, u_{\mathrm{D}}$ et $g_{\mathrm{N}}$ désignent respectivement un champ donné de vitesse et de forces surfaciques définis sur la frontière. Le tenseur de cisaillement prend la forme classique caractéristique des écoulements newtoniens :

$$
\tau(u)=\mu\left(\nabla u+\nabla u^{\mathrm{T}}\right)-\frac{2}{3} \mu \nabla \cdot u \mathrm{I}
$$

$\mu$ étant la viscosité dynamique du fluide et I le tenseur identité dans $\mathbb{R}^{d}$.

Par souci de clarté, nous débutons cet article par une description de la méthode proposée sous forme semi-discrète en temps; c'est l'objet de la section 2. Le lecteur gardera toutefois en mémoire que la formulation ainsi obtenue pour le terme de pénalisation n'est pas exactement celle qui est utilisée in fine ; pour des raisons de précision, il est en effet préférable de construire ce dernier à partir de la formulation algébrique (cf. la remarque en fin de section 4.2). La première étape du schéma ici développé est un problème elliptique pour la vitesse dont le caractère bien posé n'est pas immédiat ; nous étudions ce point dans la section 3. La discrétisation par éléments finis est ensuite décrite, ce qui nous permet de donner exactement l'algorithme utilisé (section 4). Des tests numériques pour un problème ayant une solution analytique font l'objet de la section 5 : les propriétés de convergence du schéma en temps et en espace y sont étudiées, dans le cas de conditions aux limites de Dirichlet, puis avec des conditions de Neumann sur une partie de la frontière (i.e. $\Gamma_{\mathrm{N}}$ non réduit à $\emptyset$ ).

\section{Formulation semi-discrète en temps de la méthode de pénalité-projection}

Introduisons tout d'abord quelques notations. Soit $\phi:[0, T] \times \Omega \rightarrow \mathbb{R}$ une fonction arbitraire régulière. Notons $\phi^{n}=\phi\left(t^{n}\right)$ pour $0 \leq n \leq N$. Etant donné $\phi^{0}, \ldots, \phi^{n}$, on 
obtient une approximation à l'ordre $\alpha$ de la valeur de $\phi$ à l'instant $t^{n+1}$ par extrapolation de Richardson :

$$
\phi\left(t^{n+1}\right)=\phi^{\star, n+1}+O\left(\Delta t^{\alpha}\right) \quad \text { avec } \quad \phi^{\star, n+1} \stackrel{\text { def }}{=} \sum_{j=0}^{\alpha-1} \gamma_{j} \phi^{n-j}
$$

De plus, une approximation à l'ordre $\alpha$ de la dérivée de $\phi$ à l'instant $t^{n+1}$ est donnée par la formule de différentiation rétrograde suivante :

$$
\begin{aligned}
\frac{\partial \phi}{\partial t}\left(t^{n+1}\right)=\frac{D \phi^{n+1}}{\Delta t}+ & O\left(\Delta t^{\alpha}\right) \\
& \text { avec } D \phi^{n+1} \stackrel{\text { def }}{=} \beta_{\alpha} \phi^{n+1}-\sum_{j=0}^{\alpha-1} \beta_{j} \phi^{n-j}
\end{aligned}
$$

Dans la pratique, nous utiliserons des approximations à l'ordre 1 et 2 où les coefficients $\beta_{j}$ et $\gamma_{j}$ sont donnés comme suit :

$$
\begin{array}{cccl}
\alpha=1 & D \phi^{n+1}=\phi^{n+1}-\phi^{n} & \phi^{\star, n+1}=\phi^{n} \\
\alpha=2 & D \phi^{n+1}=\frac{3}{2} \phi^{n+1}-2 \phi^{n}+\frac{1}{2} \phi^{n-1} & \phi^{\star, n+1}=2 \phi^{n}-\phi^{n-1}
\end{array}
$$

En introduisant la nouvelle variable $q=(\varrho u)$ représentant le débit massique, une semi-discrétisation linéairement implicite en temps (formellement) à l'ordre $\alpha$ du problème traité dans cette section s'écrit :

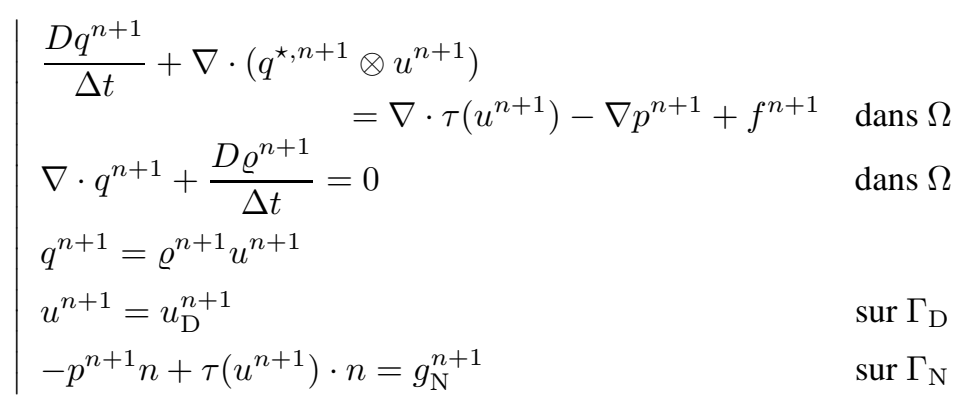

Les méthodes de projection ont pour principe d'utiliser une extrapolation $p^{\star, n+1}$ de la pression aux temps précédents dans l'équation de bilan de quantité de mouvement et les conditions aux limites associées pour obtenir une prédiction de vitesse, avant de corriger cette dernière pour vérifier le bilan de masse dans une seconde étape. 
Nous choisirons simplement ici $p^{\star, n+1}=p^{n}$. L'étape de prédiction de la méthode de pénalité-projection proposée s'écrit :

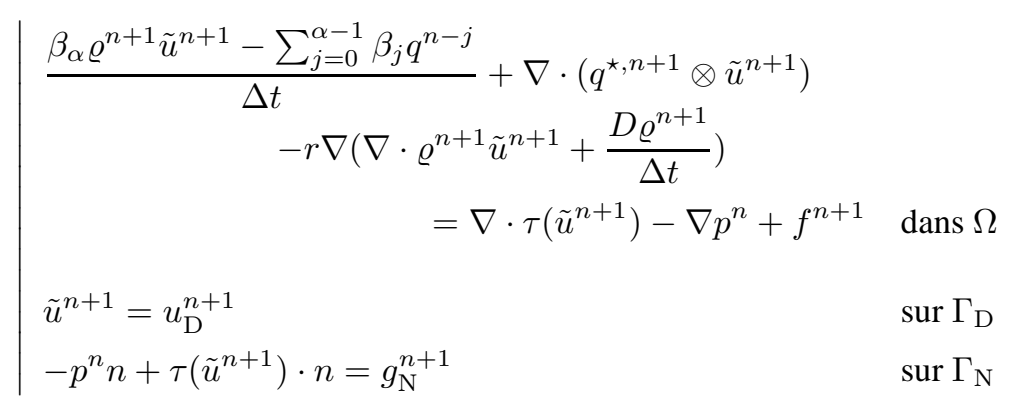

Sa spécificité réside dans l'ajout du terme de pénalisation :

$$
-r \nabla\left(\nabla \cdot \varrho^{n+1} \tilde{u}^{n+1}+\frac{D \varrho^{n+1}}{\Delta t}\right)
$$

où $r$ est un coefficient positif homogène à une viscosité que nous nommerons paramètre de pénalisation.

Ce terme est obtenu en appliquant l'opérateur gradient à l'équation de bilan de masse ; cette opération est bien sûr formelle pour le problème différentiel, mais ses analogues naturels variationnel et surtout, pour le système discret, algébrique peuvent, quant à eux, être précisément définis ; ce sera l'objet de la section 4.2.

REMARQue. [Lien avec la méthode de pénalité] - Une variante de la méthode de pénalité (Shen, 1992) consiste à changer la seconde équation du système [6] en :

$$
\frac{1}{r}\left(p^{n+1}-p^{n}\right)+\nabla \cdot q^{n+1}+\frac{D \varrho^{n+1}}{\Delta t}=0
$$

En éliminant $p^{n+1}$ entre cette relation et la première équation de [6], on obtient exactement l'étape de prédiction [7]. Par contre, la relation [9] n'est pas celle qui est utilisée ici pour incrémenter la pression.

Du fait notamment de la présence des termes de viscosité, l'inconnue naturelle de l'étape de prédiction [7] est la vitesse $\tilde{u}^{n+1}$. Il est à noter alors que, contrairement à ce qui se passe lors d'une augmentation classique (Fortin et al., 1982; Jobelin et al., 2006), le terme de pénalisation a pour contrepartie variationnelle une forme bilinéaire qui n'est ni symétrique, ni positive. Cette caractéristique pourrait être corrigée en choisissant, à la place du terme proposé, l'expression suivante :

$$
-r \varrho^{n+1} \nabla\left(\nabla \cdot \varrho^{n+1} \tilde{u}^{n+1}+\frac{D \varrho^{n+1}}{\Delta t}\right)
$$

Ce dernier choix présente l'inconvénient majeur que, ne pouvant plus s'écrire comme le gradient d'une quantité, il ne s'incorporera pas naturellement, par la suite, dans 
l'incrément de pression. En outre, nous prouvons dans la section suivante que, pour un pas de temps plus petit qu'une valeur seuil $\Delta t_{0}$ indépendante du paramètre de pénalisation $r$, l'équation aux dérivées partielles [7] admet une solution et une seule, ce qui, d'une certaine manière, répond aux interrogations que soulève la non-positivité du terme de pénalisation choisi.

Pour compléter l'algorithme, il convient maintenant de construire l'étape de projection. Soit $H$ l'espace affine défini comme suit :

$$
H=\left\{q \in \mathrm{L}(\Omega)^{d}, \quad \nabla \cdot q=-\frac{D \varrho^{n+1}}{\Delta t}, \quad q \cdot n=\varrho^{n+1} u_{\mathrm{D}} \cdot n \operatorname{sur} \Gamma_{\mathrm{D}}\right\}
$$

La seconde étape revient alors à effectuer une projection $\mathrm{L}^{2}$-orthogonale du débit prédit $\varrho^{n+1} \tilde{u}^{n+1}$ sur $H$; par un argument de décomposition de Hodge, on obtient alors le système suivant :

$$
\mid \begin{array}{ll}
\beta_{\alpha} \frac{q^{n+1}-\varrho^{n+1} \tilde{u}^{n+1}}{\Delta t}+\nabla \phi=0 & \text { dans } \Omega \\
\nabla \cdot q^{n+1}=-\frac{D \varrho^{n+1}}{\Delta t} & \text { dans } \Omega \\
q^{n+1} \cdot n=\varrho^{n+1} u_{\mathrm{D}} \cdot n & \operatorname{sur} \Gamma_{\mathrm{D}}
\end{array}
$$

En prenant la divergence de la première équation et en utilisant la seconde, on obtient le problème elliptique suivant pour $\phi$ :

$$
\Delta \phi=\frac{\beta_{\alpha}}{\Delta t}\left(\nabla \cdot \varrho^{n+1} \tilde{u}^{n+1}+\frac{D \varrho^{n+1}}{\Delta t}\right)
$$

auquel il convient d'adjoindre des conditions aux limites. Sur les frontières où la vitesse est fixée, on a :

$$
q^{n+1} \cdot n=\varrho^{n+1} u_{\mathrm{D}}^{n+1} \cdot n=\varrho^{n+1} \tilde{u}^{n+1} \cdot n
$$

et par conséquent :

$$
\nabla \phi \cdot n=0 \quad \operatorname{sur} \Gamma_{\mathrm{D}}
$$

Sur les frontières de Neumann $\Gamma_{N}$ (ou frontières ouvertes), la condition aux limites provient de la condition de $\mathrm{L}^{2}$-orthogonalité de la projection sur $H$. Cette dernière s'écrit :

$$
\int_{\Omega}\left(q^{n+1}-\varrho^{n+1} \tilde{u}^{n+1}\right) \cdot\left(q^{n+1}-v\right)=0 \quad \forall v \in H
$$

Grâce à la première relation de [10] que l'on intègre par parties, puis par définition de $H$, on a :

$$
\begin{aligned}
\forall v \in H, \quad 0 \quad & =\int_{\Omega} \nabla \phi \cdot\left(q^{n+1}-v\right) \\
& =\int_{\Gamma_{\mathrm{N}}} \phi\left(q^{n+1}-v\right) \cdot n-\int_{\Omega} \phi \nabla \cdot\left(q^{n+1}-v\right) \\
& =\int_{\Gamma_{\mathrm{N}}} \phi\left(q^{n+1}-v\right) \cdot n
\end{aligned}
$$


Cette relation nous donne la condition aux limites de $\phi$ sur la frontière $\Gamma_{\mathrm{N}}$ :

$$
\phi=0 \quad \operatorname{sur} \Gamma_{\mathrm{N}}
$$

En rassemblant les relations obtenues, l'inconnue $\phi$ est solution du problème suivant :

$$
\mid \begin{array}{ll}
\Delta \phi=\frac{\beta_{\alpha}}{\Delta t}\left(\nabla \cdot \varrho^{n+1} \tilde{u}^{n+1}+\frac{D \varrho^{n+1}}{\Delta t}\right) & \operatorname{dans} \Omega \\
\nabla \phi \cdot n=0 & \text { sur } \Gamma_{\mathrm{D}} \\
\phi=0 & \text { sur } \Gamma_{\mathrm{N}}
\end{array}
$$

Une fois $\phi$ calculée, la première relation de [10] permet de réactualiser le débit :

$$
q^{n+1}=\varrho^{n+1} \tilde{u}^{n+1}-\frac{\Delta t}{\beta_{\alpha}} \nabla \phi \quad \text { dans } \Omega
$$

Enfin, si l'on somme cette même relation avec l'équation de prédiction [7], on obtient :

$$
\begin{aligned}
& \frac{\beta_{\alpha} q^{n+1}-\sum_{j=0}^{\alpha-1} \beta_{j} q^{n-j}}{\Delta t}+\nabla \cdot\left(q^{\star, n+1} \otimes \tilde{u}^{n+1}\right) \\
& \quad=\nabla \cdot \tau\left(\tilde{u}^{n+1}\right)-\nabla\left[p^{n}-r\left(\nabla \cdot \varrho^{n+1} \tilde{u}^{n+1}+\frac{D \varrho^{n+1}}{\Delta t}\right)+\phi\right]+f^{n+1}
\end{aligned}
$$

Cette relation n'est rien d'autre que la reconstitution de l'équation de quantité de mouvement, ce qui suggère l'expression suivante pour la pression en fin de pas :

$$
p^{n+1}=p^{n}-r\left(\nabla \cdot \varrho^{n+1} \tilde{u}^{n+1}+\frac{D \varrho^{n+1}}{\Delta t}\right)+\phi
$$

En conclusion, effectuer un pas de temps consiste à résoudre en séquence les problèmes elliptiques [7] et [11] puis à réactualiser le débit et la pression par respectivement [12] et [13].

\section{Sur le caractère bien posé de l'étape de prédiction}

L'étude présentée dans cette section est motivée par le fait qu'il est important dans la pratique de pouvoir choisir le paramètre de pénalisation $r$ indépendamment du pas de temps ; nous confirmons que la méthode de pénalité-projection satisfait cette propriété, dans la mesure où l'étape de prédiction constitue un problème bien posé, pour un pas de temps inférieur à un pas de temps seuil qui est bien indépendant de $r$. Ce résultat n'est pas immédiat, du fait que des estimations a priori inhabituelles doivent être prouvées, pour deux raisons : d'une part, le champ advectif n'est pas à divergence nulle, et la forme bilinéaire associée au terme convectif n'est en conséquence pas antisymétrique; d'autre part, le terme de pénalisation n'est pas symétrique.

A des fins de simplifications, nous supposerons que des conditions aux limites de Dirichlet homogènes sont imposées à la vitesse sur la totalité de la frontière $\Gamma$ (i.e. 
$\Gamma_{\mathrm{D}}=\Gamma, u_{\mathrm{D}}=0$ et $\left.\Gamma_{\mathrm{N}}=\emptyset\right)$. Le problème que nous étudions ici prend alors la forme suivante :

$$
\text { Trouver } u \in \mathrm{H}_{0}^{1}(\Omega)^{d} \text { tel que } \quad a(u, v)=(f, v), \quad \forall v \in \mathrm{H}_{0}^{1}(\Omega)^{d}
$$

où, pour une discrétisation du premier ordre en temps :

$$
\begin{aligned}
a(u, v) & =\frac{1}{\Delta t} \int_{\Omega} \varrho u \cdot v+\int_{\Omega} \nabla \cdot(q \otimes u) \cdot v+c(u, v) \\
& +r \int_{\Omega} \nabla \cdot \varrho u \nabla \cdot v
\end{aligned}
$$

Le passage à une discrétisation du second ordre de la dérivée temporelle n'aurait d'autre effet que de multiplier le premier terme par un coefficient positif constant, et l'adaptation des arguments développés ici ne nécessiterait que des modifications mineures.

Le débit $q$ et la masse volumique $\varrho$ sont connus; nous supposons que $q$ est borné dans $\mathrm{L}^{\infty}(\Omega)^{d}$ et que $\varrho$ vérifie d'une part que $0<\varrho_{\min } \leq \varrho \leq \varrho_{\max }$, d'autre part que $\nabla \varrho$ est borné dans $\mathrm{L}^{\infty}(\Omega)^{d}$. Il est à noter que ces deux hypothèses font que $\nabla \varrho / \varrho$ est également borné dans $\mathrm{L}^{\infty}(\Omega)^{d}$, propriété qui sera utilisée par la suite.

La forme bilinéaire $c(\cdot, \cdot)$ correspond à la dissipation visqueuse. Pour l'exprimer, nous supposons que la viscosité est constante, si bien que la divergence du tenseur de cisaillement s'écrit, pour une fonction régulière :

$$
\nabla \cdot \tau(u)=\mu \Delta u+\frac{1}{3} \mu \nabla \nabla \cdot u
$$

et, en conséquence :

$$
c(u, v)=\mu \int_{\Omega} \nabla u: \nabla v+\frac{1}{3} \mu \int_{\Omega} \nabla \cdot u \nabla \cdot v
$$

Nous débutons cette section par deux lemmes techniques.

Lemme 1 - L'opérateur de convection vérifie les résultats de continuité suivants :

$$
\begin{aligned}
& \forall u \in \mathrm{H}_{0}^{1}(\Omega)^{d}, \\
& \quad\left|\int_{\Omega} \nabla \cdot(q \otimes u) \cdot \varrho u\right| \\
& \quad \leq \sqrt{d}\|q\|_{\infty}\left(1+\sqrt{d} c_{\mathrm{p}}\left\|\frac{\nabla \varrho}{\varrho}\right\|_{\infty}\right)\|\nabla u\|_{0}\|\varrho u\|_{0} \\
& \quad\left|\int_{\Omega} \nabla \cdot\left(q \otimes \frac{1}{\varrho} u\right) \cdot u\right| \leq \sqrt{d}\left\|\frac{1}{\varrho} q\right\|_{\infty}\|\nabla u\|_{0}\|u\|_{0}
\end{aligned}
$$

où $c_{\mathrm{p}}=c_{\mathrm{p}}(\Omega)$ désigne la constante de Poincaré. 
Preuve - Du fait que le champ de vitesse $u$ s'annule aux frontières du domaine, nous avons :

$$
\begin{aligned}
\int_{\Omega} \nabla \cdot(q \otimes u) \cdot \varrho u & =-\int_{\Omega} q \otimes u: \nabla(\varrho u) \\
& =-\int_{\Omega} \sum_{i, j=1}^{d} q_{i} u_{j} \frac{\partial}{\partial x_{j}}\left(\varrho u_{i}\right) \\
& =-\underbrace{\int_{\Omega} \sum_{i, j=1}^{d} q_{i} \varrho u_{j} \frac{\partial u_{i}}{\partial x_{j}}}_{(1)}-\underbrace{\int_{\Omega} \sum_{i, j=1}^{d} q_{i} \varrho u_{j} \frac{1}{\varrho} \frac{\partial \varrho}{\partial x_{j}}}_{(2)} u_{i}
\end{aligned}
$$

En appliquant l'inégalité de Cauchy-Schwarz d'abord dans $\mathbb{R}^{d \times d}$ puis dans $\mathrm{L}^{2}$, la première de ces deux intégrales se majore comme suit :

$$
\begin{aligned}
|(1)| & \leq \int_{\Omega}\left[\sum_{i, j=1}^{d}\left(q_{i} \varrho u_{j}\right)^{2}\right]^{1 / 2}\left[\sum_{i, j=1}^{d}\left(\frac{\partial u_{i}}{\partial x_{j}}\right)^{2}\right]^{1 / 2} \\
& \leq\left[\int_{\Omega} \sum_{i, j=1}^{d}\left(q_{i} \varrho u_{j}\right)^{2}\right]^{1 / 2}\left[\int_{\Omega} \sum_{i, j=1}^{d}\left(\frac{\partial u_{i}}{\partial x_{j}}\right)^{2}\right]^{1 / 2} \\
& \leq \sqrt{d}\|q\|_{\infty}\|\varrho u\|_{0}\|\nabla u\|_{0}
\end{aligned}
$$

De la même manière, nous avons pour la seconde intégrale :

$$
\begin{aligned}
|(2)| & \leq\left[\int_{\Omega} \sum_{i, j=1}^{d}\left(q_{i} \varrho u_{j}\right)^{2}\right]^{1 / 2}\left[\int_{\Omega} \sum_{i, j=1}^{d}\left(\frac{1}{\varrho} \frac{\partial \varrho}{\partial x_{j}} u_{i}\right)^{2}\right]^{1 / 2} \\
& \leq \sqrt{d}\|q\|_{\infty}\|\varrho u\|_{0} \sqrt{d}\left\|\frac{\nabla \varrho}{\varrho}\right\|_{\infty}\|u\|_{0} \\
& \leq d c_{\mathrm{p}}\|q\|_{\infty}\left\|\frac{\nabla \varrho}{\varrho}\right\|_{\infty}\|\varrho u\|_{0}\|\nabla u\|_{0}
\end{aligned}
$$

En regroupant ces deux inégalités, on obtient la première relation à démontrer. Pour la seconde, on procède de même :

$$
\int_{\Omega} \nabla \cdot\left(q \otimes \frac{1}{\varrho} u\right) \cdot u=-\int_{\Omega}\left(q \otimes \frac{1}{\varrho} u\right): \nabla u=\int_{\Omega} \sum_{i, j=1}^{d} \frac{1}{\varrho} q_{i} u_{j} \frac{\partial u_{i}}{\partial x_{j}}
$$

Par l'inégalité de Cauchy-Schwarz, il vient :

$$
\left|\int_{\Omega} \nabla \cdot\left(q \otimes \frac{1}{\varrho} u\right) \cdot u\right| \leq\left[\int_{\Omega} \sum_{i, j=1}^{d}\left(\frac{q_{i}}{\varrho} u_{j}\right)^{2}\right]^{1 / 2}\left[\int_{\Omega} \sum_{i, j=1}^{d}\left(\frac{\partial u_{i}}{\partial x_{j}}\right)^{2}\right]^{1 / 2}
$$


et l'inégalité recherchée s'en déduit en faisant apparaître $\left\|\frac{1}{\varrho} q\right\|_{\infty}$ dans le premier terme.

Lemme 2 - L'opérateur de diffusion vérifie les inégalités de stabilité suivantes :

$$
\begin{aligned}
\forall u \in \mathrm{H}_{0}^{1}(\Omega)^{d}, & \\
c(u, \varrho u) \geq \quad & \frac{1}{2} \mu \varrho_{\min }\|\nabla u\|_{0}^{2}+\frac{1}{6} \mu \varrho_{\min }\|\nabla \cdot u\|_{0}^{2} \\
& -\frac{2 d}{3} \frac{\mu}{\varrho_{\min }}\left\|\frac{\nabla \varrho}{\varrho}\right\|_{\infty}^{2}\|\varrho u\|_{0}^{2} \\
c\left(\frac{1}{\varrho} u, u\right) \geq \quad & \frac{1}{2} \frac{\mu}{\varrho_{\max }}\|\nabla u\|_{0}^{2}+\frac{1}{6} \frac{\mu}{\varrho_{\max }}\|\nabla \cdot u\|_{0}^{2} \\
& -\frac{2 d}{3} \mu \varrho_{\max }\left\|\nabla\left(\frac{1}{\varrho}\right)\right\|_{\infty}^{2}\|u\|_{0}^{2}
\end{aligned}
$$

Preuve - Nous avons :

$$
c(u, \varrho u)=\mu \int_{\Omega} \nabla u: \nabla(\varrho u)+\frac{\mu}{3} \int_{\Omega} \nabla \cdot u \nabla \cdot \varrho u
$$

En développant les dérivées des produits, il vient :

$$
c(u, \varrho u)=\mu \int_{\Omega} \varrho\|\nabla u\|^{2}+T_{1}+\frac{\mu}{3} \int_{\Omega} \varrho(\nabla \cdot u)^{2}+T_{2}
$$

avec :

$$
\begin{aligned}
& T_{1}=\mu \int_{\Omega} \sum_{i, j=1}^{d} \frac{1}{\varrho} \frac{\partial \varrho}{\partial x_{j}} \frac{\partial u_{i}}{\partial x_{j}} \varrho u_{i} \\
& T_{2}=\frac{1}{3} \mu \int_{\Omega}\left(\sum_{i=1}^{d} \frac{\partial u_{i}}{\partial x_{i}}\right)\left(\sum_{i=1}^{d} \frac{1}{\varrho} \frac{\partial \varrho}{\partial x_{i}} \varrho u_{i}\right)
\end{aligned}
$$

Le premier de ces deux termes est majoré en utilisant l'inégalité de Cauchy-Schwarz successivement dans $\mathbb{R}^{d \times d}$ et dans $\mathrm{L}^{2}$ comme suit :

$$
\begin{aligned}
\left|T_{1}\right| & \leq \mu\left\|\frac{\nabla \varrho}{\varrho}\right\|_{\infty} \int_{\Omega} \sum_{i, j=1}^{d} \frac{\partial u_{i}}{\partial x_{j}} \varrho u_{i} \\
& \leq \mu\left\|\frac{\nabla \varrho}{\varrho}\right\|_{\infty} \int_{\Omega}\left[\sum_{i, j=1}^{d}\left(\frac{\partial u_{i}}{\partial x_{j}}\right)^{2}\right]^{1 / 2}\left[d \sum_{i=1}^{d}\left(\varrho u_{i}\right)^{2}\right]^{1 / 2} \\
& \leq \mu\left\|\frac{\nabla \varrho}{\varrho}\right\|_{\infty}\left[\int_{\Omega} \sum_{i, j=1}^{d}\left(\frac{\partial u_{i}}{\partial x_{j}}\right)^{2}\right]^{1 / 2}\left[d \int_{\Omega} \sum_{i=1}^{d}\left(\varrho u_{i}\right)^{2}\right]^{1 / 2} \\
& \leq \sqrt{d} \mu\left\|\frac{\nabla \varrho}{\varrho}\right\|_{\infty}\|\nabla u\|_{0}\|\varrho u\|_{0}
\end{aligned}
$$


De la même manière, par l'inégalité de Cauchy-Schwarz dans $L^{2}$, nous avons pour le second terme :

$$
\begin{aligned}
\left|T_{2}\right| & \leq \frac{1}{3} \mu\left\|\frac{\nabla \varrho}{\varrho}\right\|_{\infty}\left[\int_{\Omega}\left(\sum_{i=1}^{d} \frac{\partial u_{i}}{\partial x_{i}}\right)^{2}\right]^{1 / 2}\left[\int_{\Omega}\left(\sum_{i=1}^{d} \varrho u_{i}\right)^{2}\right]^{1 / 2} \\
& \leq \frac{\sqrt{d}}{3} \mu\left\|\frac{\nabla \varrho}{\varrho}\right\|_{\infty}\|\nabla \cdot u\|_{0}\|\varrho u\|_{0}
\end{aligned}
$$

L'équation [14] a donc pour conséquence :

$$
\begin{aligned}
& c(u, \varrho u) \geq \mu \varrho_{\min }\|\nabla u\|_{0}^{2}+\frac{\mu}{3} \varrho_{\min }\|\nabla \cdot u\|_{0}^{2} \\
&-\sqrt{d} \mu\left\|\frac{\nabla \varrho}{\varrho}\right\|_{\infty}\|\varrho u\|_{0}\left(\|\nabla u\|_{0}+\frac{1}{3}\|\nabla \cdot u\|_{0}\right)
\end{aligned}
$$

et la première inégalité recherchée s'en déduit par l'inégalité de Young.

Pour la seconde inégalité, on a :

$$
c\left(\frac{1}{\varrho} u, u\right)=\mu \int_{\Omega} \frac{1}{\varrho}\|\nabla u\|^{2}+T_{1}+\frac{\mu}{3} \int_{\Omega} \frac{1}{\varrho}(\nabla \cdot u)^{2}+T_{2}
$$

avec :

$$
\begin{aligned}
& T_{1}=\mu \int_{\Omega} \sum_{i, j=1}^{d} \frac{\partial}{\partial x_{j}}\left(\frac{1}{\varrho}\right) u_{i} \frac{\partial u_{i}}{\partial x_{j}} \\
& T_{2}=\frac{1}{3} \mu \int_{\Omega}\left[\nabla\left(\frac{1}{\varrho}\right) \cdot u\right] \nabla \cdot u
\end{aligned}
$$

Le premier de ces deux termes se majore comme suit :

$$
\begin{aligned}
\left|T_{1}\right| & \leq \mu\left\|\nabla\left(\frac{1}{\varrho}\right)\right\|_{\infty}\left[\int_{\Omega} \sum_{i, j=1}^{d}\left(\frac{\partial u_{i}}{\partial x_{j}}\right)^{2}\right]^{1 / 2}\left[\int_{\Omega} \sum_{i, j=1}^{d} u_{i}^{2}\right]^{1 / 2} \\
& \leq \sqrt{d} \mu\left\|\nabla\left(\frac{1}{\varrho}\right)\right\|_{\infty}\|u\|_{0}\|\nabla u\|_{0}
\end{aligned}
$$

De même, pour le second :

$$
\left|T_{2}\right| \leq \frac{1}{3} \sqrt{d} \mu\left\|\nabla\left(\frac{1}{\varrho}\right)\right\|_{\infty}\|u\|_{0}\|\nabla \cdot u\|_{0}
$$

Le résultat recherché s'en déduit en reportant ces deux relations dans [15] et en utilisant l'inégalité de Young.

On rappelle le résultat général suivant, introduit dans (Nečas, 1962) (ou, par exemple, (Ern et al., 2002, théorème 3.2.3), pour une présentation récente). 
Théorème 1 - Soit $V$ un espace de Hilbert muni du produit scalaire $(\cdot, \cdot)$ et de la norme associée $\|\cdot\|, a(\cdot, \cdot)$ une forme bilinéaire sur $V \times V$ et $f \in V$. On suppose que les deux hypothèses suivantes sont vérifiées :

$$
\begin{aligned}
& \inf _{w \in V} \sup _{v \in V} \frac{a(w, v)}{\|w\|\|v\|} \geq \beta>0 \\
& (\forall w \in V, a(w, v)=0) \Longrightarrow(v=0)
\end{aligned}
$$

Alors le problème :

Trouver $u \in V$ tel que $a(u, v)=(f, v) \quad \forall v \in V$

admet une solution et une seule.

Ce théorème est exploité pour démontrer le résultat suivant.

Proposition - Si le pas de temps $\Delta t$ est plus petit qu'une valeur seuil $\Delta t_{0}$ indépendante du paramètre de pénalisation $r$ (plus précisément, tel que les inégalités [17] et [18] soient vérifiées), l'étape de prédiction admet une solution et une seule.

Preuve - Nous allons vérifier les deux hypothèses du théorème 1 . Soit donc $u$ une fonction de $\mathrm{H}_{0}^{1}(\Omega)^{d}$. Par les premières relations des deux lemmes ci-avant, nous avons :

$$
\begin{aligned}
& a(u, \varrho u) \geq \\
& \frac{1}{\Delta t}\|\varrho u\|_{0}^{2}-\sqrt{d}\|q\|_{\infty}\left(1+\sqrt{d} c_{\mathrm{p}}\left\|\frac{\nabla \varrho}{\varrho}\right\|_{\infty}\right)\|\nabla u\|_{0}\|\varrho u\|_{0} \\
& +\frac{1}{2} \mu \varrho_{\min }\|\nabla u\|_{0}^{2}+\frac{1}{6} \mu \varrho_{\min }\|\nabla \cdot u\|_{0}^{2}-\frac{2 d}{3} \frac{\mu}{\varrho_{\min }}\left\|\frac{\nabla \varrho}{\varrho}\right\|_{\infty}^{2}\|\varrho u\|_{0}^{2} \\
& +r\|\nabla \cdot \varrho u\|_{0}^{2}
\end{aligned}
$$

En majorant le second terme du membre de droite par l'inégalité de Young, il vient :

$$
\begin{aligned}
& a(u, \varrho u) \geq \\
& {\left[\frac{1}{\Delta t}-\frac{d}{\mu \varrho_{\min }}\|q\|_{\infty}^{2}\left(1+\sqrt{d} c_{\mathrm{p}}\left\|\frac{\nabla \varrho}{\varrho}\right\|_{\infty}\right)^{2}\right.} \\
& \left.-\frac{2 d}{3} \frac{\mu}{\varrho_{\min }}\left\|\frac{\nabla \varrho}{\varrho}\right\|_{\infty}^{2}\right]\|\varrho u\|_{0}^{2} \\
& +\frac{1}{4} \mu \varrho_{\min }\|\nabla u\|_{0}^{2}+\frac{1}{6} \mu \varrho_{\min }\|\nabla \cdot u\|_{0}^{2}+r\|\nabla \cdot \varrho u\|_{0}^{2}
\end{aligned}
$$

Compte tenu du fait que, par hypothèse sur $\varrho$ :

$$
\|\varrho u\|_{0}^{2} \geq \varrho_{\min }^{2}\|u\|_{0}^{2} \quad \text { et } \quad\|\varrho u\|_{1} \leq c(\varrho)\|u\|_{1}
$$


l'inégalité ci-avant fournit la première relation du théorème 1 , pourvu que $\Delta t$ soit suffisamment petit pour que la condition suivante soit vérifiée :

$$
\frac{1}{\Delta t}-\frac{d}{\mu \varrho_{\min }}\|q\|_{\infty}^{2}\left(1+\sqrt{d} c_{\mathrm{p}}\left\|\frac{\nabla \varrho}{\varrho}\right\|_{\infty}\right)^{2}-\frac{2 d}{3} \frac{\mu}{\varrho_{\min }}\left\|\frac{\nabla \varrho}{\varrho}\right\|_{\infty}^{2}>0 \text { [17] }
$$

De la même manière, en utilisant cette fois-ci les secondes relations des lemmes 1 et 2 , on a :

$$
\begin{aligned}
a\left(\frac{1}{\varrho} u, u\right) & \geq \frac{1}{\Delta t}\|u\|_{0}^{2}-\sqrt{d}\left\|\frac{1}{\varrho} q\right\|_{\infty}\|\nabla u\|_{0}\|u\|_{0} \\
& +\frac{1}{2} \frac{\mu}{\varrho_{\max }}\|\nabla u\|_{0}^{2}+\frac{1}{6} \frac{\mu}{\varrho_{\max }}\|\nabla \cdot u\|_{0}^{2} \\
& -\frac{2 d}{3} \mu \varrho_{\max }\left\|\nabla\left(\frac{1}{\varrho}\right)\right\|_{\infty}^{2}\|u\|_{0}^{2}+r\|\nabla \cdot u\|_{0}^{2}
\end{aligned}
$$

Soit, toujours par l'inégalité de Young :

$$
\begin{aligned}
a\left(\frac{1}{\varrho} u, u\right) & \geq\left[\frac{1}{\Delta t}-d \frac{\varrho_{\max }}{\mu}\left\|\frac{1}{\varrho} q\right\|_{\infty}^{2}-\frac{2 d}{3} \mu \varrho_{\max }\left\|\nabla\left(\frac{1}{\varrho}\right)\right\|_{\infty}^{2}\right]\|u\|_{0}^{2} \\
& +\frac{1}{4} \frac{\mu}{\varrho_{\max }}\|\nabla u\|_{0}^{2}+\frac{1}{6} \frac{\mu}{\varrho_{\max }}\|\nabla \cdot u\|_{0}^{2}+r\|\nabla \cdot u\|_{0}^{2}
\end{aligned}
$$

ce qui fournit la deuxième relation du théorème 1 , pourvu que :

$$
\frac{1}{\Delta t}-d \frac{\varrho_{\max }}{\mu}\left\|\frac{1}{\varrho} q\right\|_{\infty}^{2}-\frac{2 d}{3} \mu \varrho_{\max }\left\|\nabla\left(\frac{1}{\varrho}\right)\right\|_{\infty}^{2}>0
$$

REMARQUE. - En fait, la seconde hypothèse du théorème 1 peut être démontrée directement à partir de la relation [16], en changeant $u$ par $u / \varrho$; on obtient alors, sous la condition [17] :

$$
\forall u \in \mathrm{H}_{0}^{1}(\Omega)^{d}, \quad a\left(\frac{1}{\varrho} u, u\right) \geq c\|u\|_{0}^{2}
$$

où $c$ est une constante strictement positive, ce qui permet de conclure. Demander au pas de temps de vérifier [18] n'est donc pas nécessaire; en revanche, nous obtenons sous cette hypothèse un résultat plus fort, à savoir une stabilité en norme $\mathrm{H}^{1}$ :

$$
\forall u \in \mathrm{H}_{0}^{1}(\Omega)^{d}, \quad a\left(\frac{1}{\varrho} u, u\right) \geq c\left(\|u\|_{0}^{2}+\|\nabla u\|_{0}^{2}\right)
$$

\section{Une implémentation éléments finis}

L'objet de cette section est d'effectuer une description des schémas introduits dans cet article sous forme algébrique. Nous présentons tout d'abord la formulation variationnelle du problème obtenu après une semi-discrétisation en temps semi-implicite 
couplant bilan de masse et de quantité de mouvement, ainsi qu'une méthode de résolution de ce problème. Dans un second temps, nous établissons la forme algébrique de la méthode de pénalité-projection qui, nous le rappelons, est celle utilisée en pratique et diffère légèrement, pour le terme de pénalisation, de ce que l'on obtiendrait si l'on discrétisait en espace les équations du schéma semi-discret en temps présenté dans la section 2.

\subsection{Le schéma linéairement implicite}

Nous supposons donnés deux espaces éléments finis de Lagrange $V_{h}$ et $M_{h}$, le premier étant utilisé pour discrétiser respectivement vitesse et débit, le second pour la pression, et inclus respectivement dans les espaces $\mathrm{H}^{1}(\Omega)^{d}$ et $\mathrm{L}^{2}(\Omega)$. On notera :

$$
V_{h}^{\Gamma_{\mathrm{D}}}=\left\{v \in V_{h}, v=0 \operatorname{sur} \Gamma_{\mathrm{D}}\right\}
$$

Une fois la discrétisation en temps effectuée, le problème revient à rechercher à chaque pas de temps $n+1$, la vitesse $u_{h}^{n+1} \in V_{h}$, la pression $p_{h}^{n+1} \in M_{h}$ et le débit $q_{h}^{n+1} \in$ $V_{h}$.

Nous décomposons alors la vitesse et le débit en deux parties telles que :

$$
u_{h}=u_{\mathrm{D}}+u_{\mathrm{F}}, \quad q_{h}=q_{\mathrm{D}}+q_{\mathrm{F}}
$$

où $u_{\mathrm{F}}$ (respectivement $q_{\mathrm{F}}$ ) appartient à l'espace $V_{h}^{\Gamma_{\mathrm{D}}}$ et est le champ de vitesse (respectivement débit) inconnu. Les fonctions $u_{\mathrm{D}}$ et $q_{\mathrm{D}}$ peuvent être considérées comme des relèvements discrets des conditions aux limites de Dirichlet non homogènes. La formulation variationnelle du précédent problème au pas de temps $n+1$ s'écrit :

$$
\begin{aligned}
& \text { Trouver }\left(u_{\mathrm{F}}^{n+1}, q_{\mathrm{F}}^{n+1}\right) \in\left(V_{h}^{\Gamma_{\mathrm{D}}}\right)^{2} \text { et } p_{h}^{n+1} \in M_{h} \text { tels que, } \forall v \in V_{h}^{\Gamma_{\mathrm{D}}}, \\
& \forall \varphi \in M_{h}: \\
& \qquad \begin{array}{c}
\int_{\Omega} \frac{\varrho^{n+1} \beta_{\alpha}\left(u_{\mathrm{F}}^{n+1}+u_{\mathrm{D}}^{n+1}\right)-\sum_{j=0}^{\alpha-1} \beta_{j} q_{h}^{n-j}}{\Delta t} \cdot v \\
+\int_{\Omega} \nabla \cdot\left(q_{h}^{\star, n+1} \otimes\left(u_{\mathrm{F}}^{n+1}+u_{\mathrm{D}}^{n+1}\right)\right) \cdot v \\
+\int_{\Omega} \tau\left(u_{\mathrm{F}}^{n+1}+u_{\mathrm{D}}^{n+1}\right): \nabla v-\int_{\Omega} p_{h}^{n+1} \nabla \cdot v \\
\int_{\Omega}\left(\frac{D \varrho^{n+1}}{\partial t}+\nabla \cdot\left[\varrho^{n+1}\left(u_{\mathrm{F}}^{n+1}+u_{\mathrm{D}}^{n+1}\right)\right]\right) \varphi=0 \\
\int_{\Omega}\left(q_{\mathrm{F}}^{n+1}+q_{\mathrm{D}}^{n+1}\right) \cdot v=\int_{\Omega} \varrho^{n+1}\left(u_{\mathrm{F}}^{n+1}+u_{\mathrm{D}}^{n+1}\right) \cdot v
\end{array}
\end{aligned}
$$


En exploitant de la manière usuelle cette formulation variationnelle, nous obtenons le système algébrique suivant :

$$
\mid \begin{aligned}
& \frac{\beta_{\alpha}}{\Delta t} \mathbf{M}_{\varrho^{n+1}} \mathbf{U}_{\mathbf{F}}+\mathbf{A} \mathbf{U}_{\mathbf{F}}+\mathbf{B}^{\mathrm{T}} \mathbf{P}=\mathbf{F} \\
& \mathbf{B}_{\varrho^{n+1}} \mathbf{U}_{\mathbf{F}}=\mathbf{G} \\
& \mathbf{M} \mathbf{Q}_{\mathbf{F}}=\mathbf{M}_{\varrho^{n+1}} \mathbf{U}_{\mathbf{F}}+\mathbf{H}
\end{aligned}
$$

où, par souci de simplicité, les exposants relatifs à l'avancée en temps ont été omis. Les vecteurs d'inconnues $\mathbf{U}_{\mathbf{F}}$ et $\mathbf{P}$ correspondent à la vitesse $u_{\mathrm{F}}^{n+1}$ et à la pression $p_{h}^{n+1}$ en fin de pas de temps. Les opérateurs discrets sont les suivants : $\mathbf{M}$ et $\mathbf{M}_{\varrho^{n+1}}$ désignent respectivement la matrice de masse de vitesse standard et pondérée par la masse volumique à $t^{n+1}\left(\right.$ i.e. $\left.\varrho^{n+1}\right)$, $\mathbf{A}$ regroupe convection et diffusion, $\mathbf{B}_{\varrho^{n+1}}$ correspond à l'opposée de la divergence pondérée par $\varrho^{n+1}$ et $\mathbf{B}^{\mathrm{T}}$ désigne le gradient discret (donc, en conséquence, $\mathbf{B}$ correspond à l'opposé de la divergence discrète). Le second membre $\mathbf{F}$ regroupe les contributions des forces réparties, des vitesses aux pas de temps précédents et des conditions aux limites (vitesses ou contraintes imposées), $\mathbf{G}$ correspond à la dérivée en temps discrète de la masse volumique et aux conditions aux limites (vitesses imposées), tandis que $\mathbf{H}$ ne contient que la contribution de ces dernières.

Une des méthodes fréquemment utilisées pour la résolution du système correspondant dans le cas incompressible est la méthode de lagrangien augmenté (Fortin et al., 1982); nous l'extrapolons ici aux écoulements dilatables. Le principe de cet algorithme est d'ajouter un terme d'augmentation dans la première équation, puis de répéter jusqu'à convergence une séquence d'opérations consistant à résoudre cette première équation à pression fixée puis à corriger la pression.

Le terme de pénalisation est obtenu en prémultipliant l'équation de bilan de masse $\operatorname{par} \gamma \mathbf{B}^{\mathrm{T}} \mathbf{M}_{\mathbf{p}}^{-1}$, où $\gamma$ est un réel strictement positif, appelé paramètre d'augmentation et ajusté suivant le problème, et $\mathbf{M}_{\mathbf{p}}$ est une matrice de normalisation, qui peut par exemple être obtenue à partir de la matrice de masse de pression par condensation sur la diagonale.

Cet algorithme s'écrit donc sous forme algébrique de la manière suivante, en notant $k$ l'indice associé à chaque itération :

$$
\mid \begin{aligned}
& \frac{\beta_{\alpha}}{\Delta t} \mathbf{M}_{\varrho^{n+1}} \mathbf{U}_{\mathbf{F}}^{k+1}+\mathbf{A} \mathbf{U}_{\mathbf{F}}{ }^{k+1}+\gamma \mathbf{B}^{\mathrm{T}} \mathbf{M}_{\mathbf{p}}^{-1}\left(\mathbf{B}_{\varrho^{n+1}} \mathbf{U}_{\mathbf{F}}{ }^{k+1}-\mathbf{G}\right) \\
& \mathbf{P}^{k+1}=\mathbf{P}^{k}+\gamma \mathbf{M}_{\mathbf{p}}^{-1}\left(\mathbf{B}_{\varrho^{n+1}} \mathbf{U}_{\mathbf{F}}{ }^{k+1}-\mathbf{G}\right)
\end{aligned}=\mathbf{F}-\mathbf{B}^{\mathrm{T}} \mathbf{P}^{k}
$$

La pression doit être initialisée, par exemple à la pression au pas de temps précédent; la convergence est atteinte lorsque la pression n'évolue plus ou, de manière équivalente, lorsque le bilan de masse est satisfait, à une tolérance $\epsilon$ près donnée :

$$
\left\|\mathbf{B}_{\varrho^{n+1}} \mathbf{U}_{\mathbf{F}}^{k+1}-\mathbf{G}\right\|<\epsilon
$$


Une fois la vitesse de fin de pas $\mathbf{U}_{\mathbf{F}}$ obtenue, le débit est réactualisé :

$$
\mathbf{M Q}_{\mathbf{F}}=\mathbf{M}_{\varrho^{n+1}} \mathbf{U}_{\mathbf{F}}+\mathbf{H}
$$

\subsection{Méthode de pénalité-projection}

Nous allons dans cette section construire la méthode de pénalité-projection en suivant pas à pas la démarche utilisée pour établir le schéma dans le formalisme semidiscret.

En premier lieu, comme dans toute méthode de projection, on découple les équations de bilan de masse et de quantité de mouvement en explicitant la pression dans cette dernière; on obtient ainsi la relation suivante :

$$
\left(\frac{\beta_{\alpha}}{\Delta t} \mathbf{M}_{\varrho^{n+1}}+\mathbf{A}\right) \widetilde{\mathbf{U}}_{\mathbf{F}}+\mathbf{B}^{\mathrm{T}} \mathbf{P}_{\exp }=\mathbf{F}
$$

où $\mathbf{P}_{\exp }$ désigne la pression en début de pas de temps.

On obtient la première étape de l'algorithme en ajoutant à cette équation un terme de pénalisation, construit de la même manière que le terme d'augmentation introduit dans la section précédente :

$$
\left(\frac{\beta_{\alpha}}{\Delta t} \mathbf{M}_{\varrho^{n+1}}+\mathbf{A}+r \mathbf{B}^{\mathrm{T}} \mathbf{M}_{\mathbf{p}}^{-1} \mathbf{B}_{\varrho^{n+1}}\right) \widetilde{\mathbf{U}}_{\mathbf{F}}+\mathbf{B}^{\mathrm{T}} \mathbf{P}_{\exp }=\mathbf{F}+r \mathbf{B}^{\mathrm{T}} \mathbf{M}_{\mathbf{p}}^{-1} \mathbf{G}
$$

Laissée sous forme de problème de Darcy, l'étape de projection s'écrit :

$$
\mid \begin{aligned}
& \frac{\beta_{\alpha}}{\Delta t}\left(\mathbf{M} \mathbf{Q}_{\mathbf{F}}-\mathbf{M}_{\varrho^{n+1}} \widetilde{\mathbf{U}}_{\mathbf{F}}\right)+\mathbf{B}^{\mathrm{T}} \Phi=0 \\
& \mathbf{B} \mathbf{Q}_{\mathbf{F}}=\mathbf{G}
\end{aligned}
$$

En multipliant la première équation par $\mathbf{B} \mathbf{M}^{-1}$ puis en utilisant la contrainte $\mathbf{B Q}_{\mathbf{F}}=$ G, il vient :

$$
\mathbf{B M}^{-1} \mathbf{B}^{\mathrm{T}} \Phi=\frac{\beta_{\alpha}}{\Delta t}\left(\mathbf{B M}^{-1} \mathbf{M}_{\varrho^{n+1}} \tilde{\mathbf{U}}_{\mathbf{F}}-\mathbf{G}\right)
$$

Malheureusement, l'utilisation de l'inverse de $\mathbf{M}$ est coûteuse en temps calcul car, dans le cas général, cette matrice de masse n'est pas diagonale. L'équation [11] suggère alors d'approcher $\mathbf{B} \mathbf{M}^{-1} \mathbf{M}_{\varrho^{n+1}}$ par $\mathbf{B}_{\varrho^{n+1}}$ et $\mathbf{B} \mathbf{M}^{-1} \mathbf{B}^{T}$ par $\mathbf{L}$ où $\mathbf{L}$ est l'opérateur associé à un problème de Poisson avec des conditions aux limites de Dirichlet homogènes sur $\Gamma_{N}$ et de Neumann homogènes sur $\Gamma_{D}$. Cette dernière substitution n'est possible, toutefois, que pour un choix restreint d'espaces d'approximation pour la pression, excluant ceux où aucune continuité n'est requise, même en moyenne ou ponctuellement, aux interfaces entre les éléments ; l'espace utilisé dans les tests numériques présentés ici, à savoir l'élément fini de Lagrange P1 classique, est inclus dans 
$\mathrm{H}^{1}(\Omega)$. Si l'on souhaite, pour des raisons de simplicité d'implémentation, garder les mêmes dimensions pour les vecteurs $\mathbf{P}$ et $\Phi$, les conditions de Dirichlet peuvent être imposées par pénalisation :

$$
\mathbf{L}_{i j}=\int_{\Omega} \nabla \varphi_{i}^{p} \cdot \nabla \varphi_{j}^{p}+\alpha \int_{\Gamma_{N}} \varphi_{i}^{p} \cdot \varphi_{j}^{p}, \quad 1 \leq i, j \leq N_{\mathrm{dd} l}^{p}
$$

où les fonctions $\varphi_{i}^{p}$ sont les fonctions de base de l'espace d'approximation de pression $M_{h}, N_{\mathrm{ddl}}^{p}$ désigne le nombre de degrés de liberté de pression (soit la dimension de $M_{h}$ ) et le coefficient $\alpha$ est un réel positif vérifiant $\alpha \gg 1$. Enfin on réactualise la pression en fin de pas de temps:

$$
\mathbf{P}=\mathbf{P}_{\exp }+\Phi+r \mathbf{M}_{\mathbf{p}}^{-1}\left(\mathbf{B}_{\varrho^{n+1}} \widetilde{\mathbf{U}}_{\mathbf{F}}-\mathbf{G}\right)
$$

En résumé, l'algorithme décrivant un pas de temps de la méthode de pénalitéprojection est le suivant :

$$
\mid \begin{aligned}
& \left(\frac{\beta_{\alpha}}{\Delta t} \mathbf{M}_{\varrho^{n+1}}+\mathbf{A}+r \mathbf{B}^{\mathrm{T}} \mathbf{M}_{p}^{-1} \mathbf{B}_{\varrho^{n+1}}\right) \widetilde{\mathbf{U}}_{\mathbf{F}} \\
& \mathbf{L} \Phi=\frac{\beta_{\alpha}}{\Delta t}\left(\mathbf{B}_{\varrho^{n+1}} \widetilde{\mathbf{U}}_{\mathbf{F}}-\mathbf{G}\right) \quad=-\mathbf{B}^{\mathrm{T}} \mathbf{P}_{\exp }+\mathbf{F}+r \mathbf{B}^{\mathrm{T}} \mathbf{M}_{p}^{-1} \mathbf{G} \\
& \mathbf{M} \mathbf{Q}_{\mathbf{F}}=\mathbf{M}_{\varrho^{n+1}} \widetilde{\mathbf{U}}_{\mathbf{F}}-\frac{\Delta t}{\beta_{\alpha}} \mathbf{B}^{\mathrm{T}} \Phi \\
& \mathbf{P}=\mathbf{P}_{\exp }+\Phi+r \mathbf{M}_{\mathbf{p}}^{-1}\left(\mathbf{B}_{\varrho^{n+1}} \widetilde{\mathbf{U}}_{\mathbf{F}}-\mathbf{G}\right)
\end{aligned}
$$

REMARQUE. [De la nécessité de pénaliser de manière algébrique] - Une discrétisation « directe »du terme [8] écrit en semi-discret conduirait à la forme bilinéaire $c_{\text {pen }}(\cdot, \cdot)$ sur $V_{h} \times V_{h}$ suivante :

$$
c_{\mathrm{pen}}\left(u_{h}, v\right)=r \int_{\Omega}\left(\nabla \cdot \varrho u_{h}-\frac{D \varrho}{\Delta t}\right) \nabla \cdot v
$$

Or la contrainte :

$$
\nabla \cdot \varrho u_{h}-\frac{D \varrho}{\Delta t}=0
$$

ne peut être imposée à la solution discrète qu'au sens faible. Il semble donc naturel, pour ne pas voir la méthode perdre en précision pour les fortes valeurs du paramètre de pénalisation, de préférer une formulation qui utilise la contrainte réellement imposée, c'est-à-dire sa forme discrète ; c'est exactement ce qui est fait ici. Il est à noter que la formulation semi-discrète du terme de pénalisation :

$$
\nabla\left(\nabla \cdot \varrho u_{h}-\frac{D \varrho}{\Delta t}\right)
$$

est en outre incorrecte, du fait que l'intégration par parties conduisant à la forme $c_{\text {pen }}(\cdot, \cdot)$ ferait apparaître des termes de bord sur $\Gamma_{\mathrm{N}}$ qui n'ont pas lieu d'être et qui, de fait, n'ont pas leurs pendants dans la pénalisation algébrique utilisée. 


\section{Expérimentations numériques}

Nous effectuons dans cette section une comparaison entre les méthodes introduites précédemment, à savoir la méthode linéairement implicite, la méthode de pénalitéprojection et la méthode de projection incrémentale standard (obtenue à partir de la précédente en faisant $r=0$ ), en les appliquant au système suivant :

$$
\mid \begin{array}{ll}
\frac{\partial \varrho u}{\partial t}+\nabla \cdot(\varrho u \otimes u)=\mu \Delta u-\nabla p+f & \text { dans }[0,1] \times \Omega \\
\nabla \cdot \varrho u+\frac{\partial \varrho}{\partial t}=0 & \text { dans }[0,1] \times \Omega \\
u=u_{0} & \text { dans } \Omega, \text { à } t=0
\end{array}
$$

où $\Omega=] 0,1[\times] 0,1\left[, f\right.$ et $u_{0}$ sont donnés et tels que les champs de vitesse, pression et masse volumique suivants soient solution du problème :

$$
\begin{aligned}
& u(x, y, t)=\left(\begin{array}{l}
0.5 y(1-y)(2+\cos (2 \pi t)) \\
0
\end{array}\right) \\
& p(x, y, t)=-\mu(2+\cos (2 \pi t))(x-0.5) \\
& \varrho(x, y, t)=1+\frac{\left(X(x, y, t)-x_{0}\right)^{2}\left(-2 X(x, y, t)+3 x_{1}-x_{0}\right)}{\left(x_{1}-x_{0}\right)^{3}} \\
& X(x, y, t)=x-0.5 y(1-y)\left(2 t+\frac{\sin (2 \pi t)}{2 \pi}\right), \quad x_{0}=0.2 \text { et } x_{1}=0.8
\end{aligned}
$$

L'expression de la masse volumique est obtenue par la méthode des caractéristiques, en remarquant que le champ de vitesse choisi est à divergence nulle, et donc que l'équation de bilan de masse dégénère en équation de transport; cette propriété du champ de vitesse, spécifique à cette solution particulière, ne joue aucun rôle ici.

La viscosité est constante et fixée à $\mu=1$ (i.e. $\mathrm{Re} \approx 1$ ); la diffusion est donc dominante, ce qui est la situation la plus pénalisante pour ce qui concerne le traitement de conditions aux limites ouvertes par des méthodes de correction de pression, cas que nous traiterons.

Nous utilisons des éléments finis P2 pour les champs de vitesse et de débit et des éléments finis P1 pour le champ de pression; la stabilité de cette discrétisation, dite élément de Taylor-Hood, pour les problèmes incompressibles est classique (cf. par exemple (Girault et al., 1986)), elle est étendue dans le cas dilatable dans (Bernardi et al., 1992). Le maillage en triangles est obtenu en construisant tout d'abord une grille uniforme de pas $1 / 40$, puis en découpant chaque carré de la partition selon ses diagonales pour former 4 triangles isocèles. La discrétisation en temps est effectuée par une formule de différentiation rétrograde d'ordre deux, avec une estimation du champ d'advection par une extrapolation de Richardson du même ordre (équation [5]).

Les calculs présentés ici ont été réalisés avec un outil de calcul construit sur la base de la plate-forme de composants logiciels PELICANS, développée à l'IRSN (Piar, 2004). 
Dans un premier temps, nous traitons le cas où des conditions aux limites de type Dirichlet sont appliquées sur la totalité de la frontière : $u=u_{\mathrm{D}} \quad \operatorname{sur} \Gamma, \forall t \in[0,1]$ où la vitesse $u_{\mathrm{D}}$ est donnée par la solution analytique.

Les différences entre la solution numérique obtenue à $t=1$ et la solution analytique en fonction du pas de temps sont tracées sur les figures 1, 2, 3, 4, 5. Ces courbes ont l'allure usuelle : tout d'abord décroissance avec le pas de temps puis atteinte d'un plateau qui correspond à l'erreur d'approximation spatiale; ce dernier phénomène n'est observé de manière marquée que pour le débit, ce qui est très probablement dû au fait que vitesse et pression continues appartiennent toutes deux à leur espace d'approximation respectif. Tous les schémas étudiés montrent une convergence en temps approximativement d'ordre deux. Pour la méthode de projection usuelle, l'erreur de fractionnement (i.e. la différence entre la solution obtenue par la méthode de projection et la méthode linéairement implicite) est dominante à fort pas de temps : pour le débit par exemple, on constate que les erreurs associées à ces deux méthodes diffèrent d'un facteur 100. Cette erreur de fractionnement diminue avec le paramètre de pénalisation $r$, jusqu'à ce que les solutions coïncident pour $r$ grand (typiquement, $r \geq 10^{2}$ ). Ces résultats sont en accord avec ce qui est observé (Jobelin et al., 2006) ou démontré (Angot et al., 2007) pour le cas incompressible. Enfin, la comparaison des résultats obtenus pour divers maillages (non présentée ici) montre que l'erreur résiduelle obtenue pour le débit à faible pas de temps varie comme l'erreur d'approximation, à savoir comme $h^{2}$ pour la norme $\mathrm{H}^{1}$ et $h^{3}$ pour la norme $\mathrm{L}^{2}$, où $h$ est le pas de maillage.
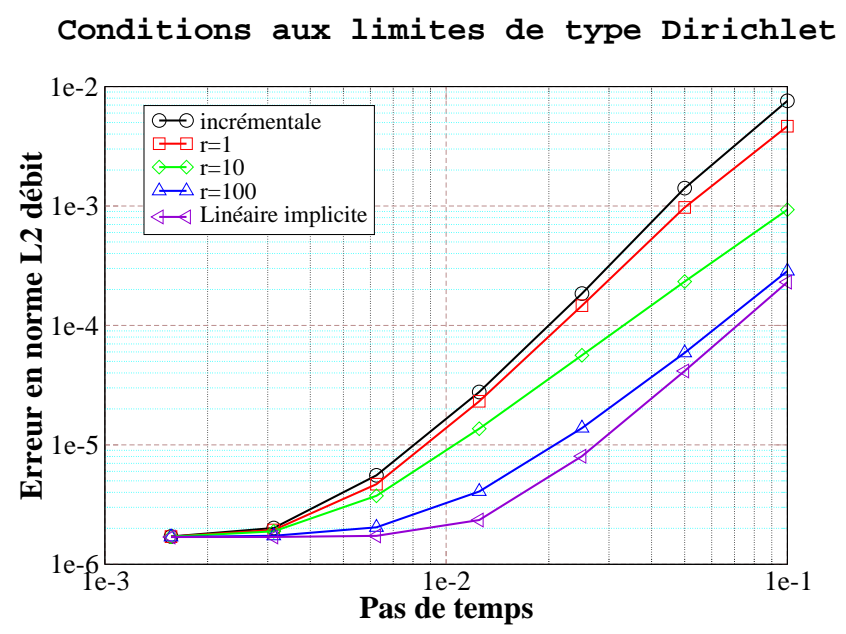

Figure 1. Cas test avec des conditions aux limites de type Dirichlet - Norme $\mathrm{L}^{2}$ de l'erreur pour le débit à $t=1$ en fonction du pas de temps pour les méthodes de projection incrémentale, pénalité-projection $(r=1, r=10, r=100)$ et linéairement implicite 

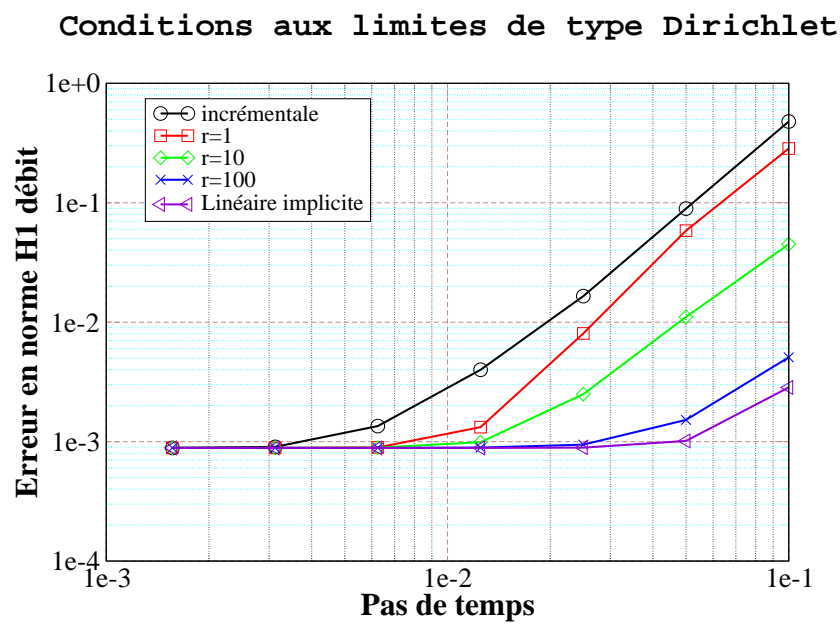

Figure 2. Cas test avec des conditions aux limites de type Dirichlet - Norme $\mathrm{H}^{1}$ de l'erreur pour le débit à $t=1$ en fonction du pas de temps pour les méthodes de projection incrémentale, pénalité-projection $(r=1, r=10, r=100)$ et linéairement implicite
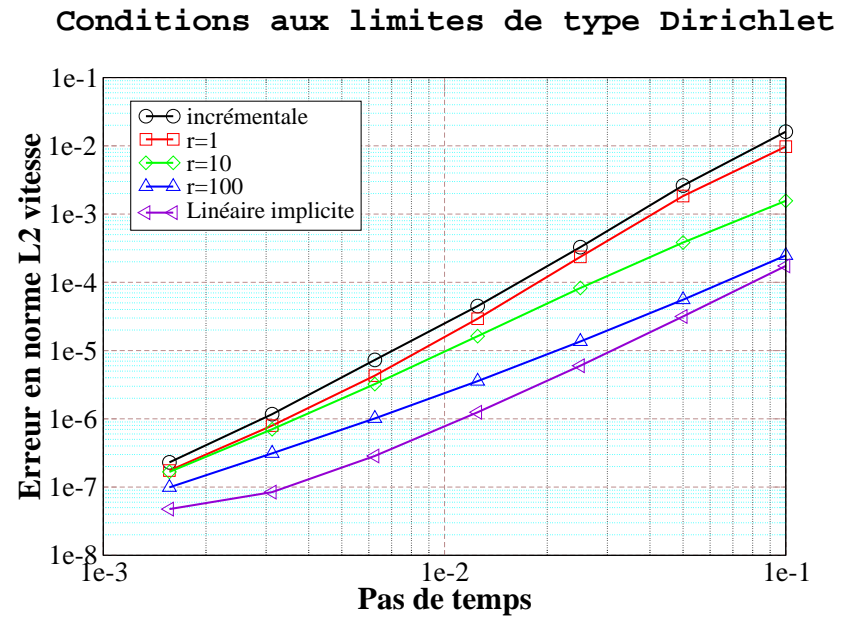

Figure 3. Cas test avec des conditions aux limites de type Dirichlet-Norme $\mathrm{L}^{2}$ de l'erreur pour la vitesse à $t=1$ en fonction du pas de temps pour les méhodes de projection incrémentale, pénalité-projection $(r=1, r=10, r=100)$ et linéairement implicite 
474 Revue européenne de mécanique numérique. Volume 17 - n 4/2008

\section{Conditions aux limites de type Dirichlet}

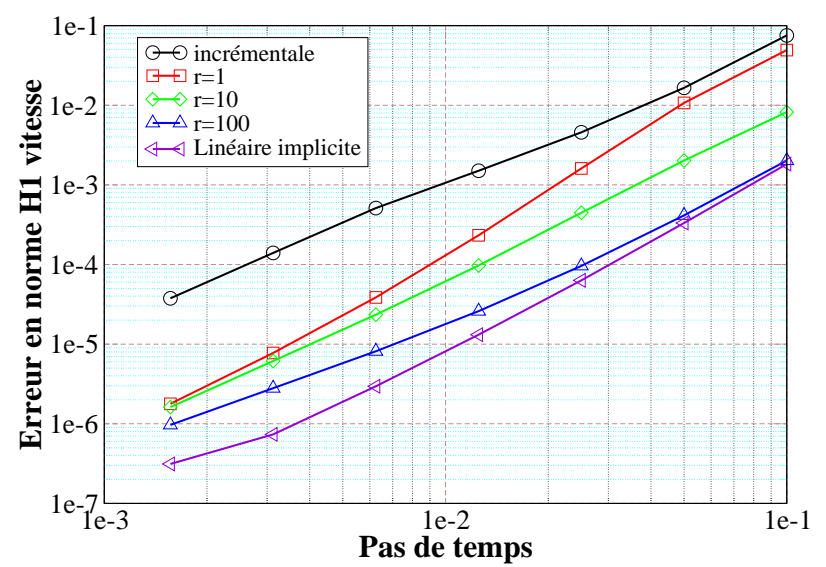

Figure 4. Cas test avec des conditions aux limites de type Dirichlet-Norme $\mathrm{H}^{1}$ de l'erreur pour la vitesse à $t=1$ en fonction du pas de temps pour les méthodes de projection incrémentale, pénalité-projection ( $r=1, r=10, r=100)$ et linéairement implicite

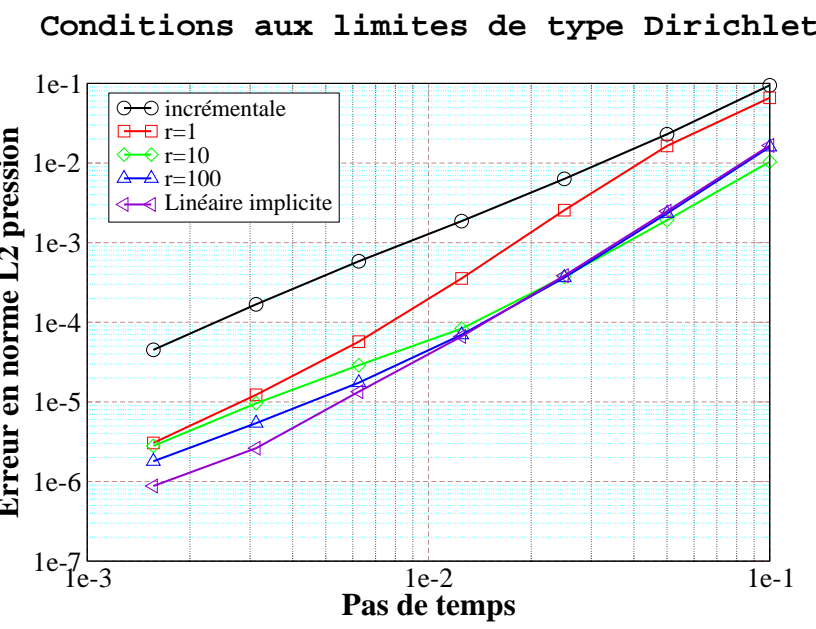

Figure 5. Cas test avec des conditions aux limites de type Dirichlet-Norme $\mathrm{L}^{2}$ de l'erreur pour la pression à $t=1$ en fonction du pas de temps pour les méthodes de projection incrémentale, pénalité-projection ( $r=1, r=10, r=100)$ et linéairement implicite 


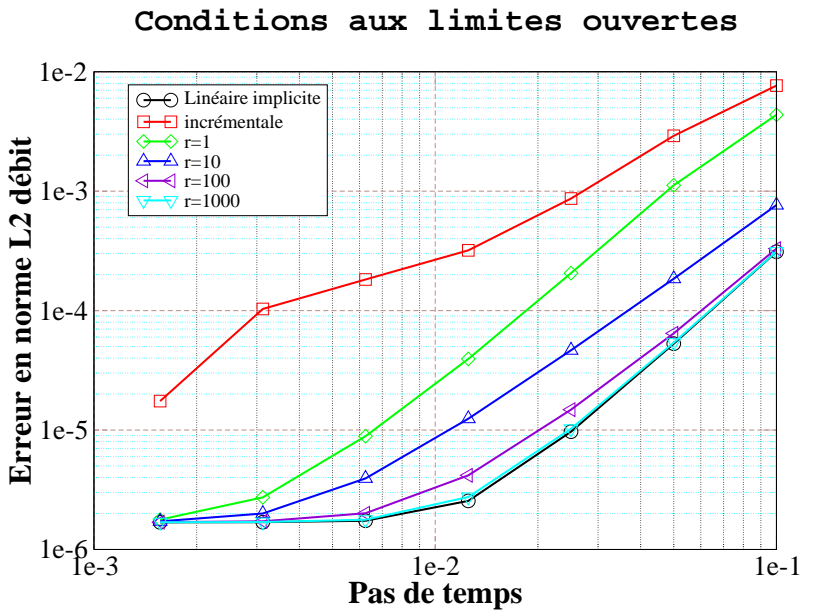

Figure 6. Cas test avec des conditions aux limites ouvertes - Norme $\mathrm{L}^{2}$ de l'erreur pour le débit à $t=1$ en fonction du pas de temps pour les méthodes de projection incrémentale, pénalité-projection ( $r=1, r=10, r=100$ ) et linéairement implicite

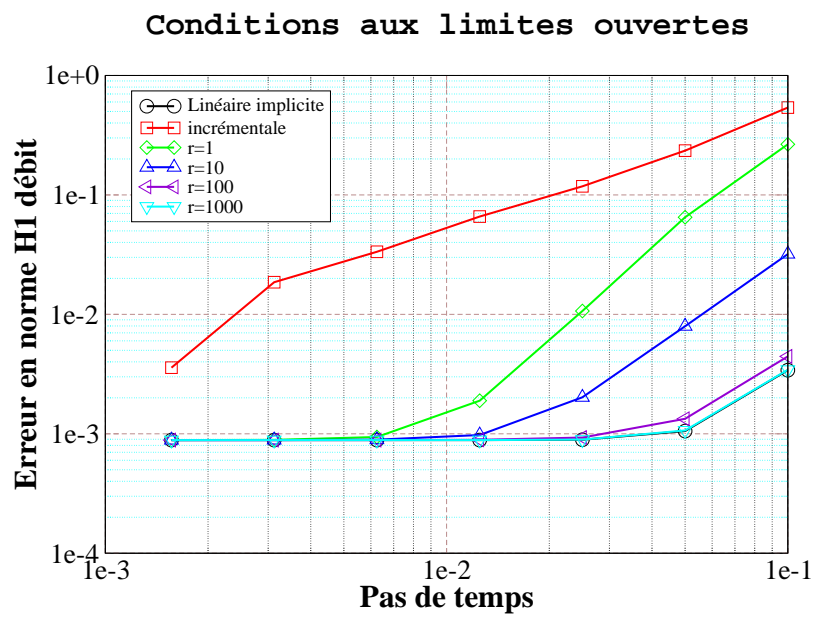

Figure 7. Cas test avec des conditions aux limites ouvertes - Norme $\mathrm{H}^{1}$ de l'erreur pour le débit à $t=1$ en fonction du pas de temps pour les méthodes de projection incrémentale, pénalité-projection ( $r=1, r=10, r=100$ ) et linéairement implicite 
476 Revue européenne de mécanique numérique. Volume 17 - n 4/2008

\section{Conditions aux limites ouvertes}

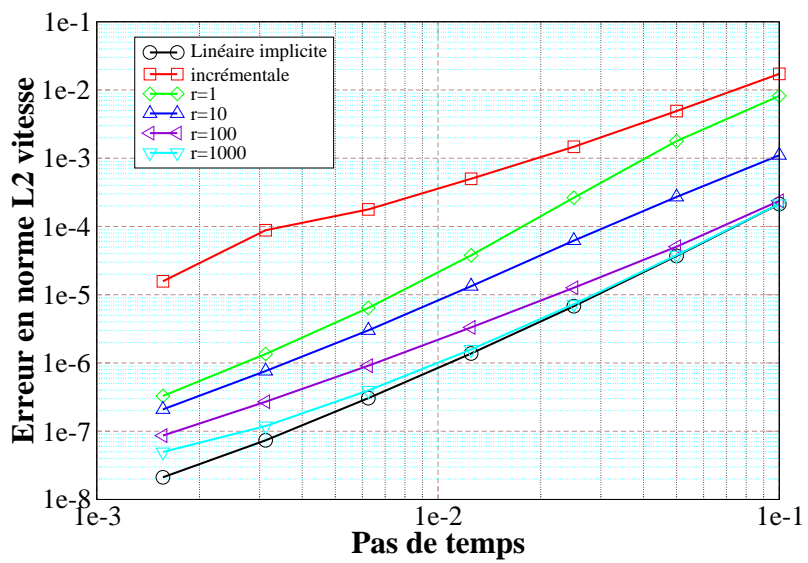

Figure 8. Cas test avec des conditions aux limites ouvertes - Norme $\mathrm{L}^{2}$ de l'erreur pour la vitesse à $t=1$ en fonction du pas de temps pour le méthodes de projection incrémentale, pénalité-projection $(r=1, r=10, r=100)$ et linéairement implicite

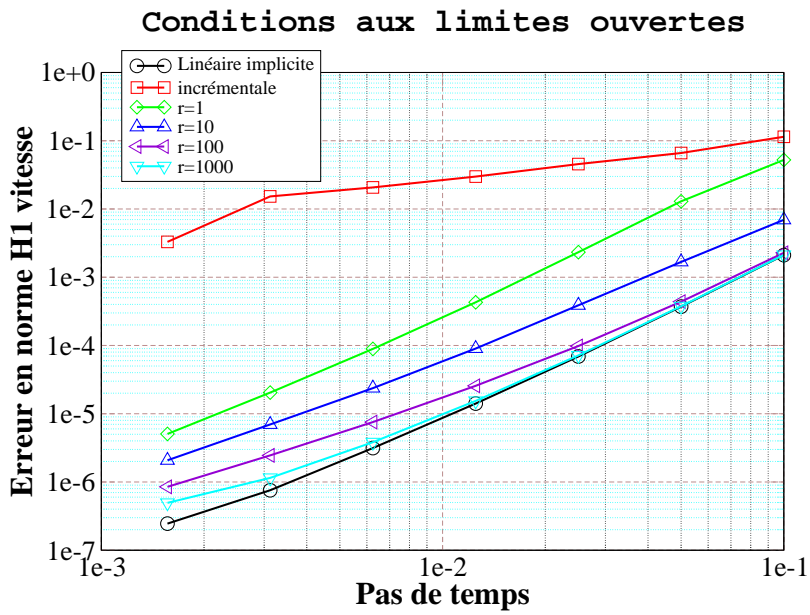

Figure 9. Cas test avec des conditions aux limites ouvertes - Norme $\mathrm{H}^{1}$ de l'erreur pour la vitesse à $t=1$ en fonction du pas de temps pour les méthodes de projection incrémentale, pénalité-projection $(r=1, r=10, r=100)$ et linéairement implicite 


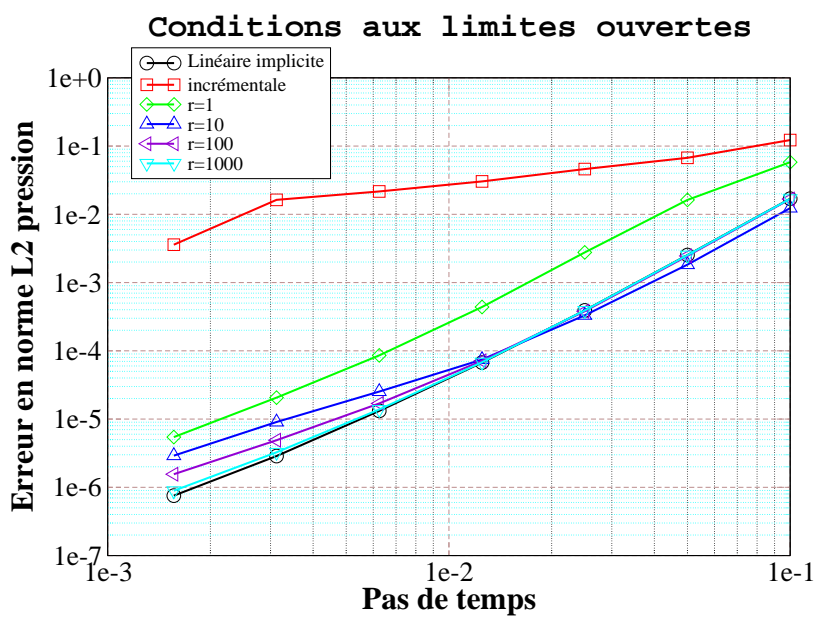

Figure 10. Cas test avec des conditions aux limites ouvertes - Norme $\mathrm{L}^{2}$ de l'erreur pour la pression à $t=1$ en fonction du pas de temps pour les méthodes de projection incrémentale, pénalité-projection ( $r=1, r=10, r=100$ ) et linéairement implicite

Nous changeons maintenant de conditions aux limites qui deviennent :

$$
\mid \begin{array}{ll}
\sigma \cdot \vec{n}=\left(\begin{array}{c}
-p_{\mathrm{D}} \\
0
\end{array}\right) & \text { sur }\{1\} \times[0 ; 1] \\
u=u(x, y, t) & \text { sur les autres frontières. }
\end{array}
$$

où la pression $p_{\mathrm{D}}$ est donnée par la solution analytique. Les résultats dans ce cas font l'objet des figures 6, 7, 8, 9, 10.

Dans le contexte des écoulements incompressibles, la méthode de projection incrémentale est connue pour perdre, en présence de conditions aux limites de Neumann (dites également « conditions aux limites ouvertes ») comme c'est le cas ici, ses propriétés de convergence. Ce phénomène est lié au fait que l'on impose, pour des raisons de stabilité, des conditions aux limites de Dirichlet à l'inconnue $\phi$ de l'étape de projection, et donc, par récurrence, compte tenu de la relation donnant l'incrément de pression pour la méthode incrémentale, à la pression elle-même. On observe alors (Guermond et al., 2005; Jobelin et al., 2006) une réduction des ordres de convergence en temps et en espace, qui prennent, probablement par hasard, les mêmes valeurs : approximativement 1 pour la vitesse en norme $\mathrm{L}^{2}$ et $1 / 2$ pour la vitesse en norme $\mathrm{H}^{1}$ et la pression en norme $L^{2}$. Ce même comportement semble se reproduire ici puis, pour les valeurs les plus faibles du pas de temps, une accélération de la convergence survient. Une étude paramétrique au pas de maillage $h$ semble montrer que le pas de temps où survient la rupture de pente dans la courbe de convergence décroît linéairement avec 
$h$, ce qui suggère l'explication suivante à ce phénomène. En exploitant des techniques d'inégalité inverse (Ciarlet, 1991), il est possible de démontrer des relations du type :

$$
\left\|u-u_{h}\right\|^{\star} \leq c(h)\left(\left\|u-u_{h}\right\|_{\star}+I_{h \star}\right)+I_{h}^{\star}
$$

où $u$ est une fonction de l'espace continu, $u_{h}$ une fonction de l'espace d'approximation, $\|\cdot\|_{\star}$ et $\|\cdot\|^{\star}$ sont deux normes, la première étant plus faible que l'autre (par exemple, norme $\mathrm{H}^{-1}$ et norme $\mathrm{L}^{2}$ ), $I_{h \star}$ et $I_{h}^{\star}$ désignent l'erreur d'interpolation respectivement en norme $\|\cdot\|_{\star}$ et en norme $\|\cdot\|^{\star}$. Imaginons que l'erreur en norme $\|\cdot\|_{\star}$ décroisse plus vite avec le pas de temps que l'erreur en norme $\|\cdot\|^{\star}$, par exemple selon les estimations suivantes (avec $\alpha<\beta$ ) :

$$
\left\|u-u_{h}\right\|^{\star} \leq c\left(\Delta t^{\alpha}+I_{h}^{\star}\right), \quad\left\|u-u_{h}\right\|_{\star} \leq c\left(\Delta t^{\beta}+I_{h \star}\right)
$$

avec $\beta>\alpha$. L'exploitation de l'inégalité [19] fournit alors une relation de la forme :

$$
\left\|u-u_{h}\right\|^{\star} \leq c^{\prime}(h)\left(\Delta t^{\beta}+I_{h \star}\right)+I_{h}^{\star}
$$

et, surtout lorsque l'erreur d'interpolation est faible, il peut exister un pas de temps seuil, dépendant de $h$, en deçà duquel cette dernière estimation est plus précise que [20]; la courbe d'erreur pourrrait alors présenter une rupture de pente (pente $\alpha$ audessus du pas de temps seuil, pente $\beta$ au-dessous). Il est à noter que de tels résultats sont classiques pour les méthodes de projection (décroissance de l'erreur de vitesse en norme $\mathrm{L}^{2}$ plus rapide qu'en norme $\mathrm{H}^{1}$, estimation d'ordre supérieur pour la pression dans une norme dépendant du maillage (Guermond, 1999)...). C'est d'ailleurs ce que l'on constate ici si l'on s'en tient à la première partie de la courbe (i.e. à fort pas de temps), puisque débit et vitesse présentent une convergence d'ordre 2 en norme $\mathrm{L}^{2}$, tandis qu'en norme $\mathrm{H}^{1}$, l'ordre tombe à 1 pour le débit et $1 / 2$ pour la vitesse ; l'ordre de convergence de la pression en norme $\mathrm{L}^{2}$ est également $1 / 2$.

Pour les méthodes autres que la projection incrémentale, les propriétés de convergence observées sont, avec des conditions aux limites ouvertes, les mêmes qu'avec des conditions de Dirichlet. On constate là encore un gain drastique de précision à fort pas de temps, dès que le paramètre de pénalisation $r$ dépasse quelques unités.

\section{Conclusion}

Nous avons décrit et étudié dans cet article une variante de la méthode de projection pour les écoulements dilatables qui en améliore considérablement la précision : l'erreur de fractionnement, dominante à fort pas de temps dès que la discrétisation en temps est formellement du second ordre, est réduite à volonté en augmentant le paramètre de pénalisation $r$; dans le cas où des conditions aux limites de Neumann sont appliquées à une partie de la frontière, la perte de convergence spatiale observée avec la méthode de projection incrémentale standard est corrigée. Malheureusement, il semble que ces propriétés soient obtenues au prix d'une détérioration du conditionnement de la matrice associée à l'étape de prédiction de vitesse, d'autant plus sensible 
que la valeur que l'on donne à $r$ est importante ; ce comportement avait été également observé pour les écoulements incompressibles (Jobelin et al., 2006). Il reste qu'à coût de calcul égal, dans les cas que nous avons étudiés, la méthode de pénalité-projection avec un paramètre de pénalisation raisonnable (typiquement, $r=10 \mu$, où $\mu$ est la viscosité) reste beaucoup plus précise que la méthode de projection incrémentale. Toutefois, le développement de solveurs performants pour l'étape de prédiction reste un sujet de recherche ouvert; l'application à ce problème de techniques multigrilles est actuellement à l'étude à l'IRSN.

\section{Bibliographie}

Angot P., Jobelin M., Latché J.-C., « Error Analysis of the Penalty-Projection Method for the Time-Dependent Stokes Equations », submitted to SIAM Journal on Numerical Analysis, 2007.

Aquilon, « Aquilon, Outil de Modélisation en Mécanique des Fluides et Transferts », 2006, http ://www.trefle.u-bordeaux1.fr/aquilon/index.html.

Babik F., Gallouët T., Latché J.-C., Suard S., Vola D., « On Some Fractional Step Schemes for Combustion Problems », Finite Volumes for Complex Applications IV (FVCA IV), Hermès, Paris, p. 505-514, 2005.

Bernardi C., Laval F., Métivet B., Pernaud-Thomas B., « Finite Element Approximation of Viscous Flows with Varying Density », SIAM Journal on Numerical Analysis, vol. 29, p. 1203$1243,1992$.

Caltagirone J.-P., Breil J., « Sur une méthode de projection vectorielle pour la résolution des équations de Navier-Stokes », Comptes-Rendus de l'Académie des Sciences, Paris - Série II, vol. 327, p. 1179-1184, 1999.

Chorin A. J., « Numerical Solution of the Navier-Stokes Equations », Mathematics of Computation, vol. 22, p. 745-762, 1968.

Ciarlet P., Finite Elements Methods - Basic Error Estimates for Elliptic Problems, vol. II of Handbook of Numerical Analysis, North-Holland, 1991.

Coré X., Angot P., Latché J.-C., « A multi-level FIC projection method for low Mach number natural convection flows », Finite Volumes for Complex Applications III (FVCA III), Éditions Hermès, Paris, p. 317-324, 2002.

Coré X., Angot P., Latché J.-C., « A multi-level local mesh refinement projection method for low Mach number flows », Mathematics and Computers in Simulation, vol. 61, p. 477-488, 2003.

Ern A., Guermond J., Eléments finis : théorie, applications, mise en cuvre, vol. 36 of Mathématiques \& Applications, Springer, 2002.

Fortin M., Glowinski R., Méthodes de Lagrangien Augmenté, Dunod, Paris, 1982.

Girault V., Raviart P.-A., Finite Element Methods for Navier-Stokes Equations. Theory and Algorithms., vol. 5 of Springer Series in Computational Mathematics, Springer-Verlag, 1986.

Goda K., « A Multistep Technique with Implicit Difference Schemes for Calculating Two- or Three-Dimensional Cavity Flows », Journal of Computational Physics, vol. 30, p. 76-95, 1979. 
Guermond J.-L., « Un résultat de convergence d'ordre deux en temps pour l'approximation des équations de Navier-Stokes par une technique de projection incrémentale », Mathematical Modelling and Numerical Analysis, vol. 33, n 1, p. 169-189, 1999.

Guermond J.-L., Shen J., « On the error estimates for the rotational pressure-correction projection methods », Mathematics of Computation, vol. 73, $\mathrm{n}^{\circ}$ 248, p. 1719-1737, 2003.

Guermond J., Minev P., Shen J., « Error Analysis of Pressure-Correction Schemes for the TimeDependent Stokes Equations with Open Boundary Conditions », SIAM Journal on Numerical Analysis, vol. 43, p. 239-258, 2005.

Guermond J., Minev P., Shen J., « An overview of Projection Methods for Incompressible Flows », Computer Methods in Applied Mechanics and Engineering, vol. 195, p. 60116045, 2006.

Jobelin M., Méthodes de Projection pour le Calcul d'Ecoulements Incompressibles ou Dilatables, Thèse de doctorat, Université de Marseille II, 2006.

Jobelin M., Lapuerta C., Latché J.-C., Angot P., Piar B., « A Finite Element Penalty-Projection Method for Incompressible Flows », Journal of Computational Physics, vol. 217, p. 502518, 2006.

Majda A., Sethian J., « The Derivation and Numerical Solution of the Equations for Zero Mach Number Solution », Combustion Science and Techniques, vol. 42, p. 185-205, 1985.

Nečas J., «Sur une méthode pour résoudre les équations aux dérivées partielles de type elliptique, voisine de la variationnelle », Ann. Scuola Norm. Sup. Pisa, vol. 16, p. 305-326, 1962.

Piar B., PELICANS : Un Outil d'Implémentation de Solveurs d'Équations aux Dérivées Partielles, Note Technique n² 2004/33, IRSN/DPAM/SEMIC, 2004.

Shen J., « On Error Estimates of some Higher Order Projection and Penalty-Projection Methods for Navier-Stokes Equations », Numerische Mathematik, vol. 62, p. 49-73, 1992.

Temam R., « Sur l'Approximation de la Solution des Équations de Navier-Stokes par la Méthode des pas Fractionnaires (II) », Archive for Rational Mechanics and Analysis, vol. 33, p. 377-385, 1969.

Timmermans L., Minev P., Van de Vosse F., « An approximate projection scheme for incompressible flow using spectral elements », International Journal for Numerical Methods in Fluids, vol. 22, p. 673-688, 1996.

Van Kan J., « A Second-Order Accurate Pressure-Correction Scheme for Viscous Incompressible Flow », SIAM Journal on Scientific and Statistical Computing, vol. 7, n 3, p. 870-891, 1986. 